\title{
Image Preprocessing Assessment Detecting Low Contrast Regions under Non-homogeneous Light Conditions
}

\author{
Camilo Vargas ${ }^{1}$, Jeyson Molina ${ }^{1}$, John W. Branch ${ }^{1}$, and Alejandro Restrepo ${ }^{2}$ \\ ${ }^{1}$ Escuela de Sistemas, Facultad de Minas, Universidad Nacional de Colombia Sede Medellín \\ \{cjvargas, jjmolinac, jwbranch\}@unal.edu.co \\ ${ }^{2}$ Instituto Tecnológico Metropolitano, Medellín, Colombia \\ alejandrorestrepo@itm.edu.co
}

\begin{abstract}
This paper focuses on evaluating the pre-processing impact in detecting low contrast regions on irregular surfaces with non-homogeneous lighting. Non homogeneous lighting represents an obstacle to the correct segmentation and subsequent classification of relevant image regions. For example in grayscale images, intensity variations are detected on the same region. Therefore lower contrast regions require an adequate sensitivity level at the segmentation stage. Segmentation, description and classification techniques will be applied over a set of images without pre-processing and over the same set of images with pre-processing, in order to achieve the assessment. The images used in this paper were obtained from a visual inspection prototype for flaw detection on dentures. The outcome shows that an appropriate image pre-processing is required to improve the detection process performance for the given circumstances.
\end{abstract}

Keywords: Flaw detection, automated visual inspection, low contrast, nonhomogeneous light.

\section{Introduction}

Lighting is a critical factor in image acquisition. Image acquisition over objects with irregular surfaces might cause lighting problems. This is an image information disruption related to the sample. Non homogeneous lighting is perceived in the image as a gray level intensity variation where the sample does not necessarily shows superficial changes. Besides, peculiarities such as low contrast flaws (where the segmentation algorithm cannot differentiate between the region of interest and the area that contains them) make it a nontrivial problem.

Image pre-processing seeks to ease the later detection process stages. This is gained by increasing the image definition and removing potential noise, granting a more precise segmentation results and decreasing computational cost on description and classification stages due to the noise reduction.

Several image pre-processing algorithms have been proposed in the literature in order to enhance the image and improve the overall image quality (i.e. better contrast of regions and less noise). For instance, in [1] an algorithm to improve images with 
low contrast regions is presented. The algorithm is based on the anisotropic diffusion filter which reduces image noise by performing a smoothing process that tries to preserve edges information and also tries to enhance the local contrast of different regions in the image at the same time. The filter is used to improve a subsequent segmentation process using various parameters and the result is greatly affected depending on their configuration. In this follow we take a similar approach by using an algorithm that deals with both goals (enhancing contrast and reducing the noise) in images with low contrast but using a different approach from the Anisotropic filter. We also go a step further and analyze the effect of the used pre-processing technique in the segmentation and classification processes for image object detection under nonhomogeneous light conditions. The detection process involves segmentation, description and classification stages which is applied to the same set of sample images with and without pre-processing. The outcomes from both detection processes will be compared by amount of detected flaw samples.

This paper is motivated by the need to detect low-contrast flaws on a quality assurance prototype for dentures. The prototype uses dome illumination system with an attached diffuser in order to reduce the optical effects caused by the specular and irregular dentures surfaces.

\section{$2 \quad$ Methods and Procedures}

Segmentation, description and classification techniques will be used over a set of images with and without pre-processing to carry out the detection process evaluation as shown in fig 1. Image sharpening and contrast-enhance techniques carry noise problems under the given irregular surface samples and non-homogeneous light circumstances, generating a later over-segmentation for brighter regions that appear naturally in the image. This way, we adopt a strategy that applies an image sharpening, a noise filter and finally a contrast enhance.

The techniques used for pre-processing are the unsharp mask for sharpening, total variation denoising, and finally a contrast stretching.

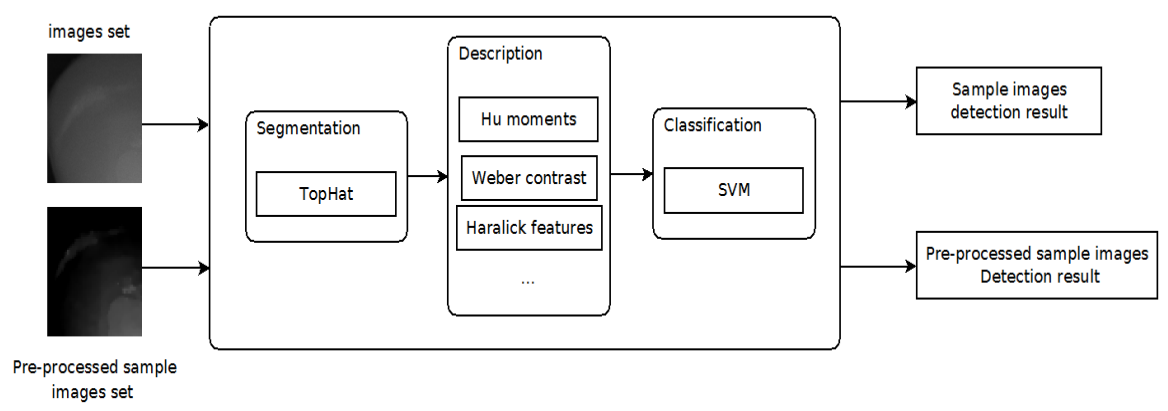

Fig. 1. Low-contrast flaws detection process diagram 


\subsection{Unsharp Mask}

Unsharp mask is an image sharpening technique. The method operates by adding a fraction of the high-pass filtered version of the input image to the original input image itself [2]. This operator is sensitive to the noise because the high-pass filter does not distinguish between the noise and the signal [3]. It can be described as shown in (1).

$$
\begin{aligned}
\hat{f}[m, n] & =\mathrm{g}[\mathrm{m}, \mathrm{n}]+\lambda H\{\mathrm{~g}[\mathrm{~m}, \mathrm{n}]\} \\
& =\mathrm{g}[\mathrm{m}, \mathrm{n}]+\lambda(\mathrm{g}[\mathrm{m}, \mathrm{n}]-L\{\mathrm{~g}[\mathrm{~m}, \mathrm{n}]\}) \\
& =(1+\lambda) \mathrm{g}[\mathrm{m}, \mathrm{n}]-\lambda L\{\mathrm{~g}[\mathrm{~m}, \mathrm{n}]\})
\end{aligned}
$$

Where $\mathrm{g}$ is the original image, $H$ represents the high-pass filter, $L$ the low-pass filter and $\hat{f}$ is the image enhancement result. Fig. 2 shows the unsharp mask process diagram.

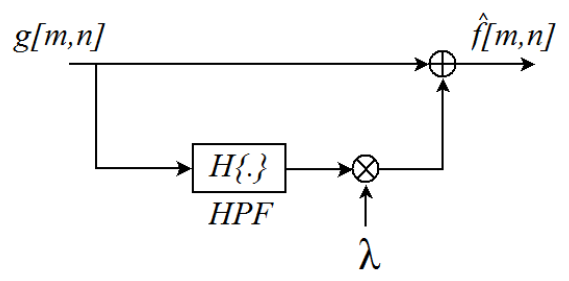

Fig. 2. Unsharp Mask process diagram

Fig. 3 shows the results of applying the unsharp mask technique over a sample image. The required input parameters are the weight $\lambda$ and the Gaussian low-pass filter sigma. Parameter selection is based on previous segmentation ROC analysis.

\subsection{Total Variation Denoising}

Total variation denoising is a noise removal technique which preserves the image edges. It is based on the principle that noise regions in an image have a high variation.

A noise image $u(x, y)$ can be described as (2):

$$
u(x, y)=u_{o}(x, y)+n(x, y)
$$

Where $u_{o}(x, y)$ represents the original image without noise and $n(x, y)$ the additive noise.

The problem is to minimize the image total variation described by (3).

$$
\int \sqrt{u_{x}^{2}+u_{y}^{2}} d x d y
$$




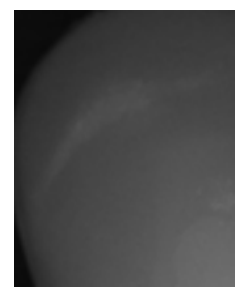

(a)

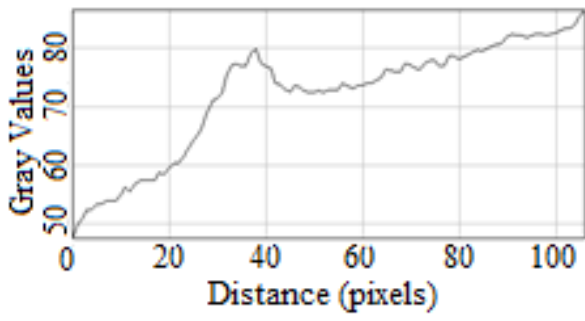

(e)

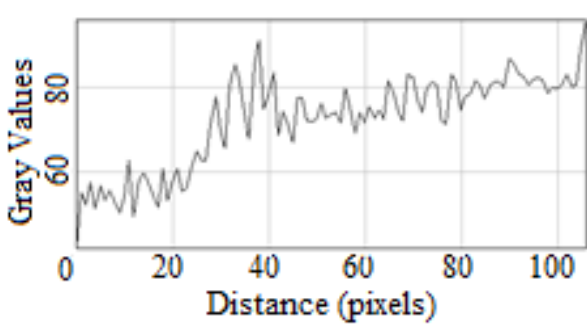

(g)

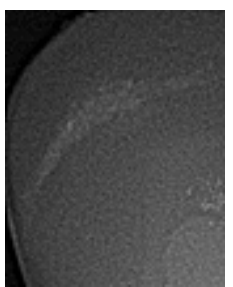

(c)

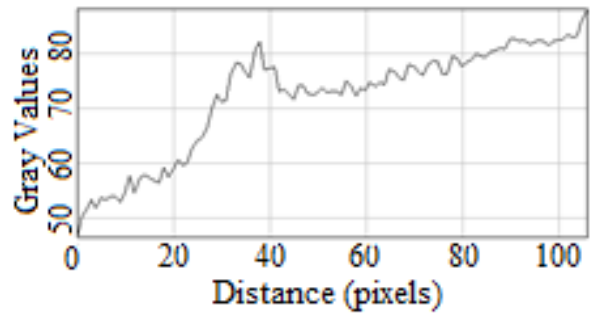

(f)

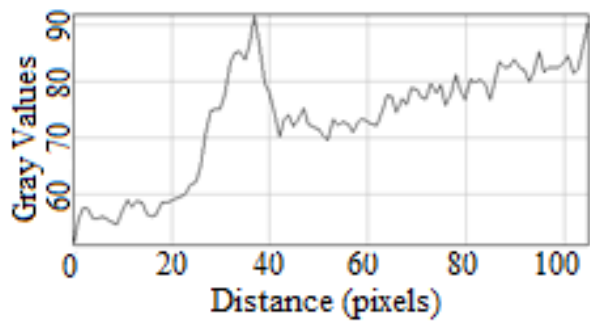

(h)

Fig. 3. Unsharp mask. Input image (a), unsharp mask result images with $\lambda=0.6$, sigma $=1(\mathrm{~b})$, $\lambda=0.9$, sigma $=1(\mathrm{c}), \lambda=0.6$, sigma $=7(\mathrm{~d})$, and their respective gray-level profile plots $(\mathrm{x}$ pixel coordinate $=40)(\mathrm{e}),(\mathrm{f}),(\mathrm{g})$ and $(\mathrm{h})$.

subject to constraints involving the mean (4) and standard deviation (5).

$$
\begin{gathered}
\int u d x d y=\int u_{0} d y d x \\
\int \frac{1}{2}\left(u-u_{0}\right)^{2} d x d y=\sigma^{2} \text { Where } \sigma^{2}>0 \text { is given. }
\end{gathered}
$$

The first constraint is related to the fact that the white noise $\mathrm{n}(\mathrm{x}, \mathrm{y})$ in (4) is zero mean. The second constraint (5) uses a priori information that the standard deviation of the noise $\mathrm{n}(\mathrm{x}, \mathrm{y})$ is $\sigma$ [4].

The algorithm used to achieve the denoising is the one introduced by [5], Fig. 4, shows the TV denoise algorithm flow diagram. It is an iterative process that estimates the input image total variation computing the L1 norm of the image's gradient (3). The image's pixels are updated according to the total variation estimation modified by 
the weight parameter and the gradient resulting in a less noisy image, where a greater weight means a greater denoising level at the expense of fidelity to input image.

The process continues until the stop criterion is met. This criterion is given by the total variation change from previous iteration to the current one. The denoise algorithm terminates if that change is lesser than eps * tv_init, where $t v_{-}$init is the input image's total variation and eps is a parameter that controls the denoise iterations (larger values translates into less iterations and less denoising). Fig. 5 shows an example of the total variation denoising used over a sample image.

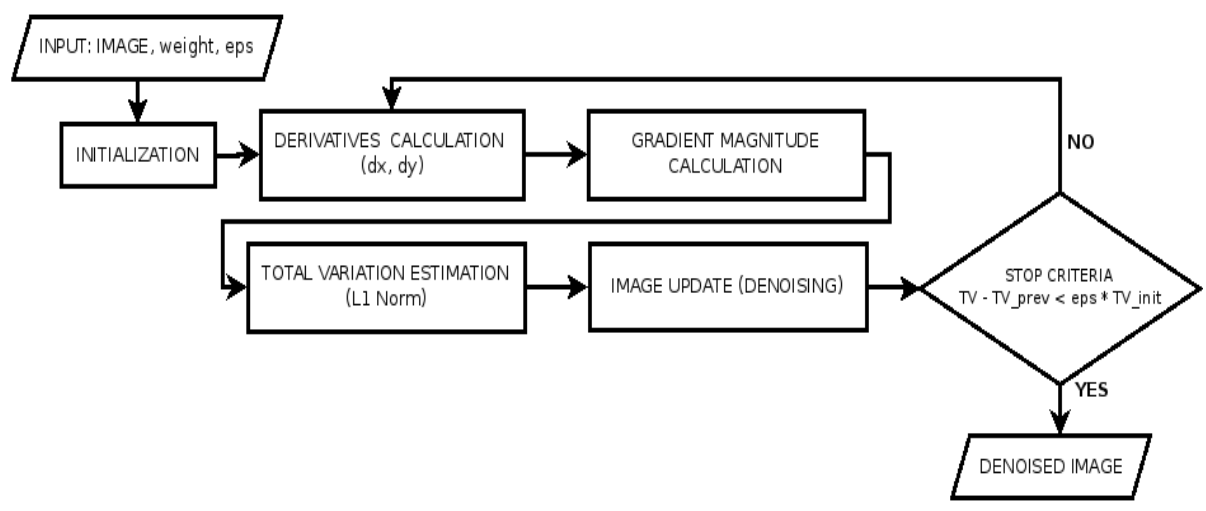

Fig. 4. TV denoise algorithm flow diagram

\subsection{Contrast Dynamic Range Stretching}

Contrast stretching is a simple contrast enhancement technique that attempts to improve the contrast in an image by stretching the range of intensity values it contains to span a desired range of values. Unlike histogram equalizing the contrast stretching result is less dramatic and tends to avoid the sometimes artificial appearance of equalized images. Applying (6) to each input pixel $P_{\text {in }}$ to obtain an output pixel $P_{\text {out }}$.

$$
P_{\text {out }}=\left(P_{\text {in }}-c\right) \frac{(b-a)}{(d-c)}+a
$$

Where $c, d$ are the maximum and minimum values of gray level in the input image, and $a, b$ are the limit values of the new range. Fig. 6, shows an example of contrast stretching result applied to a sample image and its corresponding contrast to noise ratio (CNR). CNR can be defined as (7)

$$
C N R=\frac{\text { contrast }}{\text { noise }}=\frac{2\left(s_{\text {flaw }}-s_{\text {mean }}\right)^{2}}{\sigma_{\text {flaw }}^{2}+\sigma_{\text {mean }}^{2}}
$$

Where $s$ denotes the mean and $\sigma$ denotes the standard deviation. The subscripts flaw and mean stand for the defective region and the background, respectively. 


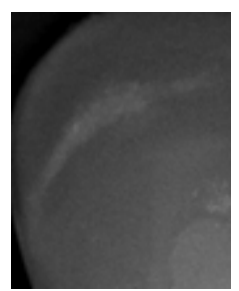

(a)

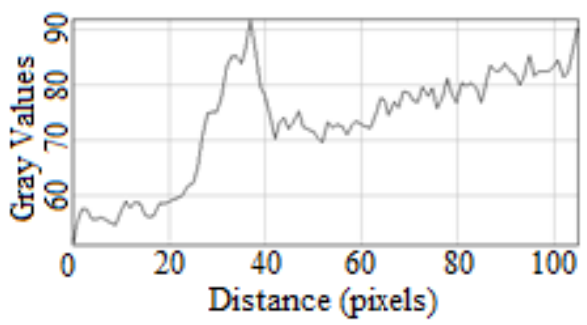

(d)

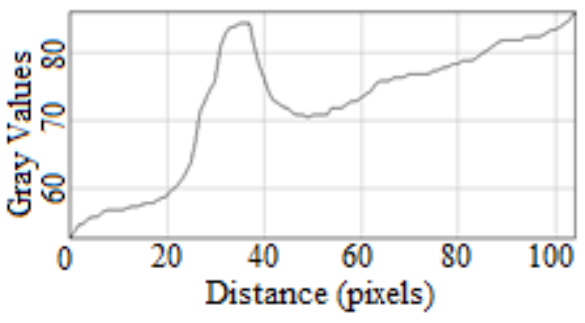

(f)

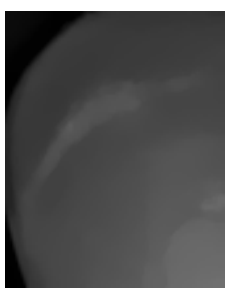

(c)

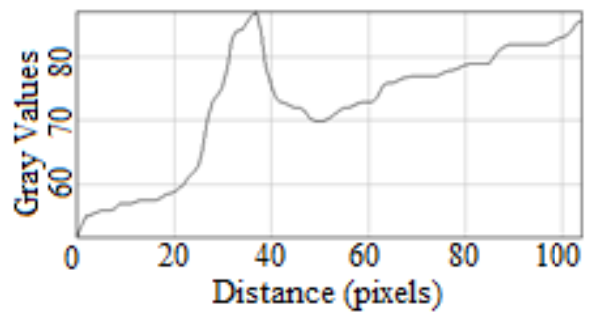

(e)

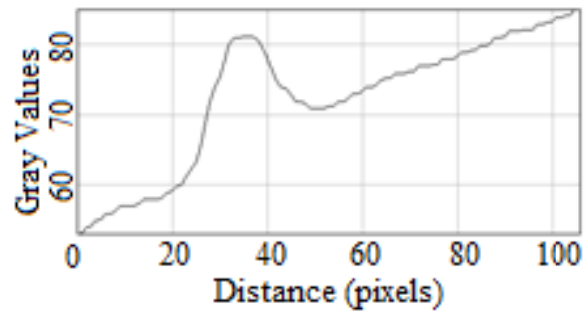

(g)

Fig. 5. Total variation denoising. Input image (a), Total variation denoising $w=5$ (b), $w=10$ $(\mathrm{c}), \mathrm{w}=30(\mathrm{~d}),($ eps $=0.0002)$. and their respective gray-level profile plots (x pixel coordinate $=$ 40) (e), (f), (g) and (h).

\subsection{Segmentation}

Segmentation consists in grouping an image in uniform regions regarding specific properties. The segmentation objective is to simplify and split the image in parts with consistent information to analyze. The morphological operation TopHat will be used to extract lighter regions concerning the area that contains them. This transform supports a threshold input parameter that allows sensitivity adjustment to low contrast changes, adding a calibrating option to face the problem.

Let $f(x)$ be the representation of the image and $b(x)$ the structuring element, where $\mathrm{B}$ is the space that $b(x)$ is defined, the transforms erosion (8) and dilate (9) are defined as follows. 


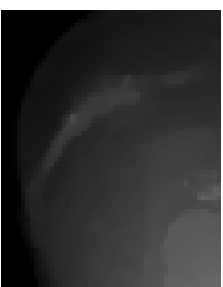

(a)

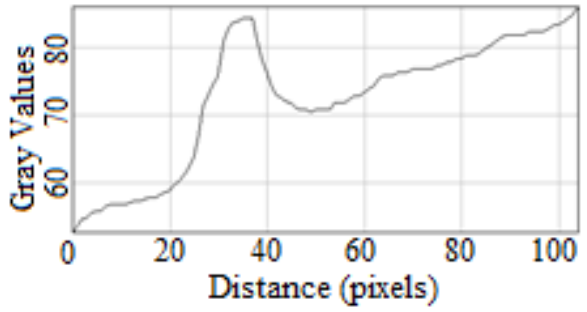

(c)

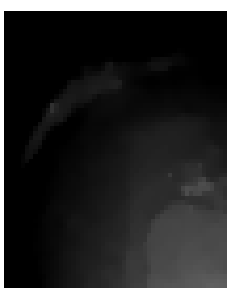

(b)

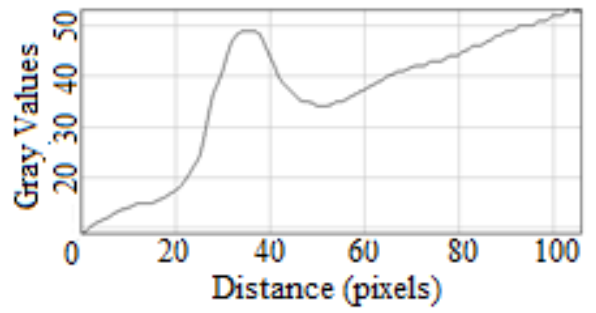

(d)

Fig. 6. Input image $C N R=0.84$ (a), contrast stretching result $C N R=0.71$ (b) $(a=40, b=210)$ and their respective gray-level profile plots $(x$ pixel coordinate $=40)(\mathrm{c}),(\mathrm{d})$

Erosion: $\quad(f \ominus b)(x)=\inf _{y \in B}\{f(y)-b(y-x)\}$

Dilation: $\quad(f \oplus b)(x)=\sup _{y \in B}\{f(y)-b(x-y)\}$

Then opening function (10) is defined as:

Opening:

$$
f \circ b=(f \ominus b) \oplus b
$$

The use of the operator opening tends to remove the lighter image region. TopHat is defined as a mathematical function for extracting lighter peaks of gray level as shown in (11).

$$
T H=\{x: f(x)-f \circ b \geq t\}
$$

Where $t$ is the threshold input parameter that allows filtering the gray level weaker peaks.

Parameters value selection is made based on a trade-off evaluation between True Positives and False Positives. Fig. 7 shows several combinations of kernel sizes $w$ and threshold values $T$ used to segment a pre-processed image. The best results (based on the selected criteria) were obtained for $w=11$ and $T=40$ after evaluating the algorithm in a sample set of preprocessed images.

\subsection{Description and Classification}

Regions obtained through segmentation are used to create a description data base. Features used for classifier training can be separated in five categories: intensity level, crossing line profile [9], geometrical, contrast and texture features. 


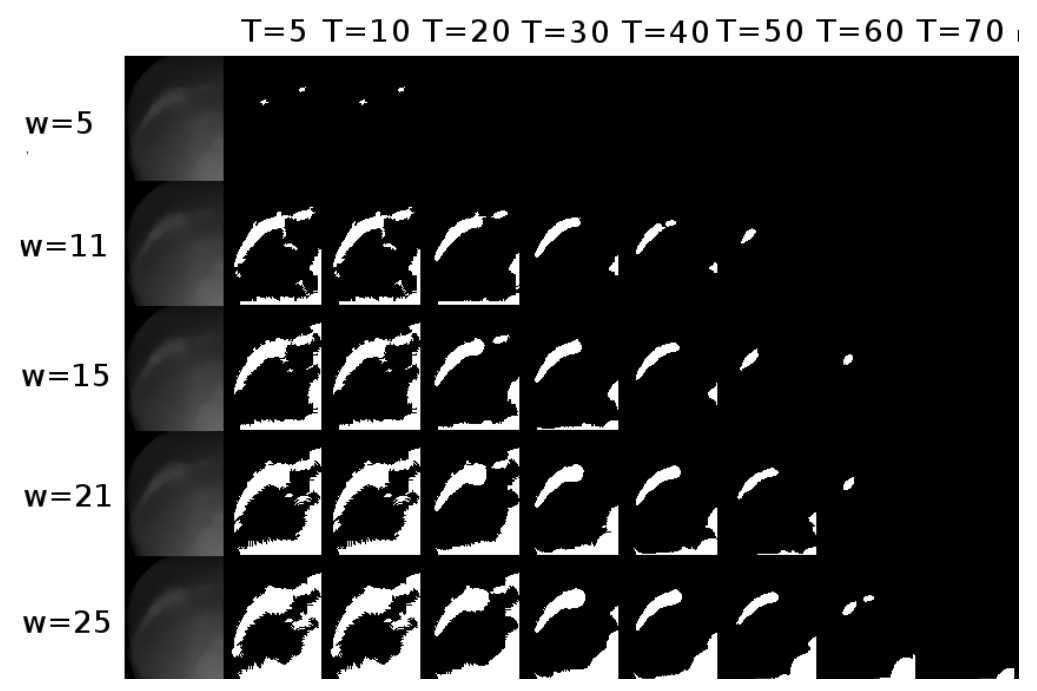

Fig. 7. Tophat segmentation evaluating several input parameter combinations

Intensity level features measures average gray level, standard deviation, $\mathrm{Hu}$ moments, among others for each region. Crossing line profile feature measures data over several lines crossing the bounding box that contains the segmented region. To obtain geometrical features, data such as area, perimeter, eccentricity, solidity and aspect ratio are extracted. Contrast features are extracted through Weber contrast, Michelson contrast and boundary average gradient. Finally, Haralick features are used to measure texture.

Once the features are extracted a principal component analysis is applied. Principal components analysis (PCA) provides an approximation to a data matrix in terms of two smaller matrixes product that captures the essential data patterns.

SMOTE (Synthetic Minority Over-sampling Technique) is an oversampling technique, where new instances from operations between neighboring samples are obtained, under the assumption that a better classifier performance can be achieved in contrast to under-sampling the majority class [7].

Since the dataset between classes is not balanced, SMOTE is applied over the obtained characteristics data base in order to balance the classes, creating flaw region instances. The instance balance process takes place after the PCA, so the characteristics selection is not made from the oversampled data base.

After the instance balance process, classifier training is done on WEKA in which support vector machines are used due to their low training cost.

A SVM (support vector machine) is an automatic learning model used in the classification and regression analysis. Input vectors are non-linearly mapped to a very highdimension feature space. In this feature space a linear decision surface or hyper plane is constructed [8]. The SVM model is generated from an initial training data from which it is able to assign a class to new samples. Confusion matrixes will be used to evaluate the SVM trained models performance. 


\section{Evaluation and Results}

The detection process is applied over a 40 defective sample images set under nonhomogeneous lighting conditions. The detection process is repeated on the 40 preprocessed images set in order to compare results. The given images set contain 73 defective regions, 2 on each sample image on average.

Table 1 presents the flaws and false alarm segmented regions obtained from the segmentation stage. The preprocessed image set shows a greater segmented regions number. It should be noted that a region marked manually as a flaw can be segmented as several unconnected smaller sub-regions.

Table 1. Number of segmented regions

\begin{tabular}{c|ccc}
\hline & $\begin{array}{c}\text { Flaw Segmented } \\
\text { regions }\end{array}$ & $\begin{array}{c}\text { False alarm re- } \\
\text { gions }\end{array}$ & $\begin{array}{c}\text { Total seg- } \\
\text { mented regions }\end{array}$ \\
\hline Original image set & 77 & 96 & 173 \\
Preprocessed image set & 127 & 363 & 490 \\
\hline
\end{tabular}

In addition, the number of non-segmented regions (false negatives) is equal to 11 and 6 for original and preprocessed images sets respectively.

From the given segmented regions the labeling and feature extraction processes are performed. Once features are extracted into a data base, a PCA is applied with maximum_atributes $=10$ and covered_variance $=0.95$ input parameters. A later SMOTE is used, generating $150 \%$ and $25 \%$ new flaw region instances from features data bases with and without pre-processing with $k$-nearest_neighbors $=5$.

A 10 fold cross validation is used to train the SVM for a radial kernel with $\varepsilon=0.001, \gamma=0.0$, coef $0=0.0$ parameters. Model training results are shown in confusion matrixes, tables 2 and 3, where the SVM trained model was re-evaluated on the original data without SMOTE.

Table 2. Confusion matrix, segmented region clasification from sample images without preprocessing

\begin{tabular}{c|cc}
\hline & False alarm region & Flaw region \\
\hline False alarm region & 95 & 1 \\
Flaw region & 3 & 74 \\
\hline
\end{tabular}

Table 3. Confusion matrix, segmented region clasification from pre-processed sample images

\begin{tabular}{c|cc}
\hline & False alarm region & Flaw region \\
\hline False alarm region & 347 & 16 \\
Flaw region & 14 & 113 \\
\hline
\end{tabular}

From the given confusion matrixes the process accuracy is calculated as shown in (12). 


$$
A c c=(T P+T N) /(T P+F P+T N+F N)
$$

The detection processes accuracy is $97.68 \%$ and $93.87 \%$ for original and preprocessed images sets respectively.

Given the amount of flaw samples and the classification results for each segmented region, the percentage of flaw samples correctly detected is calculated as shown in table 4 , where the entire inspection process performance results are presented in terms of each sample image.

Table 4. Flaw samples correctly detected

\begin{tabular}{c|ccc}
\hline & $\begin{array}{c}\text { Detected } \\
\text { flaw samples }\end{array}$ & $\begin{array}{c}\text { Undetected } \\
\text { flaw samples }\end{array}$ & $\begin{array}{c}\text { Percentage offlaw sam- } \\
\text { ples correctly detected }\end{array}$ \\
\hline Original image set & 29 & 11 & $72.5 \%$ \\
Preprocessed image set & 34 & 6 & $85 \%$ \\
\hline
\end{tabular}

This means that the detection processes have a $72.5 \%$ and $85 \%$ performance. A $12.5 \%$ increase on the overall detection process performance is noticed with the image preprocessing.

\section{Conclusion and Future Work}

A pre-processing impact assessment is presented for an automated visual low contrast flaws detection process under non-homogenous light conditions. Pre-processing becomes an important step improving definition between the low contrast regions of interest and the sample background. A higher segmented regions number and a $12.5 \%$ overall detection performance increase were noticed when pre-processing is applied.

The performance improvement is explained mainly by the definition increase on the low contrast regions of interest, which provides a higher chance to extract flaw regions at the segmentation stage and consequently a more complete later analysis.

A future optimization process will be used to adjust the used techniques input parameters, to evaluate a possible increase on the detection process performance.

\section{References}

1. Rodríguez, J.C., Molina, J., Atencio, P., Branch, J.W., Alejandro, R.: Anisotropic filtering assessment applied on superficial defects enhancement under non homogenous light conditions. Revista Avances en Sistemas e Informática 8(3), 57-62 (2011)

2. Kim, S., Allebach, J.P.: Optimal unsharp mask for image sharpening and noise removal. J. Electron. Imaging. 14(2) (2005)

3. Mahmood, N.H., Razif, M.R.M.: MTAN Gany Comparison between Median, Unsharp and Wiener filter and its effect on ultrasound stomach tissue image segmentation for Pyloric Stenosis. International Journal of Applied Science and Technology 1(5), 218-226 (2011)

4. Rudin, L.I., Osher, S., Fatemi, E.: Nonlinear total variation based noise removal algorithms. Physica D: Nonlinear Phenomena 60(1-4), 259-268 (1992) 
5. Chambolle, A.: An Algorithm for Total Variation Minimization and Applications. Journal of Mathematical Imaging and Vision 20(1-2), 89-97 (2004)

6. Wold, S., Esbensen, K., Geladi, P.: Principal Component Analysis. Chemometrics and Intelligent Laboratory Systems 2(1), 37-52 (1987)

7. Chawla, N.V., Bowyer, K.W., Hall, L.O., Kegelmeyer, W.P.: SMOTE: Synthetic Minority Over-sampling Technique. Journal of Artificial Intelligence Research 16, 321-357 (2002)

8. Cortes, C., Vapnik, V.: Support-Vector Networks. Machine Leaming 20, 273-297 (1995)

9. Mery, D.: Crossing Line Profile: A New Approach to Detecting Defects in Aluminium Die Castings. In: Bigun, J., Gustavsson, T. (eds.) SCIA 2003. LNCS, vol. 2749, pp. 725-732. Springer, Heidelberg (2003) 